\title{
Full dynamic resolution low lower DA-Converters for flat panel displays
}

\author{
C. Saas, J. Franke, and J. A. Nossek \\ Institute for Circuit Theory and Signal Processing, Munich University of Technology, Germany
}

\begin{abstract}
It has been shown that stepwise charging can reduce the power dissipated in the source drivers of a flat panel display. However the solution presented only provided a dynamic resolution of 3 bits which is not sufficient for obtaining a full color resolution display. In this work a further development of the basic idea is presented. The stepwise charging is increased to 4 bits and supplemented by a current source to provide an output signal which represents an 8 bit value with sufficient accuracy. Within this work the application is an AM-OLED flat panel display, but the concept can easily be applied to other display technologies like TFT-LCD as well.
\end{abstract}

\section{Introduction}

\subsection{Flat panel displays}

Figure 1a depicts the common architecture of all flat panel matrix displays. Although the investigations in this work have been carried out to meet the requirements of an OLED display the basic concept is as well applicable to all other types of active matrix displays. Displays that feature a selection transistor like $T_{1}$ in Fig. $1 \mathrm{~b}$ are considered to be active displays.

The proposals in this work apply to the column drivers in the display. They are as well called source drivers as the sources of the selection transistors in an active matrix display are connected to the output of this driver. This output has to provide an analog voltage value which corresponds to the intensity of a color sub-pixel. However, the row or gate drivers exhibit a digital output and are not covered in this work.

Please note that a flat panel display always needs calibration due to the nonlinear behavior of the optical element. This nonlinearity has to be calibrated at least for each new product. In high accuracy applications like pre-press every

Correspondence to: C. Saas (c@saas.de)
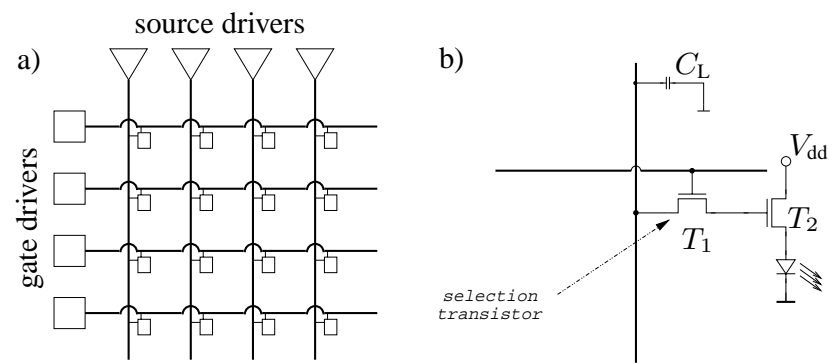

Fig. 1. (a) Architecture and (b) Pixel Schematic for an AM-OLED Display.

single display has to be calibrated every month to maintain color matching. In the proposed architecture this calibration can be realized in hardware by means of the lookup table. This solution is saving valuable dynamic resolution in the control circuitry. Please note that calibration is carried out for the whole display. Therefore variations between adjacent column lines can not be corrected. These variations can be considered small enough to not influence the image quality.

In this work a total error of $10 \%$ in the final voltage level on the column line is considered acceptable. Please note, that this small error leads to a high quality display and is not met by most consumer products on the market today.

Examinations in this work are carried out for a 1000 times 1000 pixel display, a typical load capacitance $C_{\mathrm{L}}$ of $200 \mathrm{pF}$ and an image refresh rate of $60 \mathrm{~Hz}$. The voltage range for the pixel cell has been set to $0 \mathrm{~V}$ up to $5 \mathrm{~V}$. The values chosen are exemplary for a wide range of display applications and are therefore well suited to evaluate the presented concepts.

State-of-the-art full color displays require 8 bit resolution, corresponding to $2^{8}$ possible states of brightness in each pixel cell. This dynamic range allows to distinguish between $\left(2^{8}\right)^{3}=16.7 \cdot 10^{6}$ different colors for three (RGB) cells in each pixel. 
Later calculations require the available time for charging a column line. The chosen image refresh rate corresponds to a time of $17 \mathrm{~ms}$. This defines a time of $17 \mu \mathrm{s}$ for each row. Since all columns are charged simultaneously, this is also the available time for charging one column line.

Assuming 8 bits per pixel, neglecting the data overhead and calculating one million pixels to fit into one cycle of $60 \mathrm{~Hz}$ results in pixel data running at $480 \mathrm{MHz}$, respectively a cycle time of $2 \mathrm{~ns}$ for the pixel clock.

\subsection{Stepwise Charging}

Stepwise charging is a possible realization of adiabatic switching which exhibits the advantage of not needing an oscillator. The concept of adiabatic switching has been well covered in literature (Athas et al., 2000; Ziesler et al., 2001). The necessary voltage ramps are approximated by small voltage s.pdf, which reduces the dissipated energy to

$E_{\text {diss,stepwise }}=\frac{1}{N} E_{\text {diss }}$

where $N$ is the number of s.pdf.

Please note that this holds only true if the system is given enough time to settle. Therefore, stepwise charging is like all adiabatic switching techniques a tradeoff between power and speed and most suitable in applications with limited performance requirements (Saas and Nossek, 2002).

\section{The stepwise driver}

The basic concept of how to apply stepwise charging to the source drivers in a flat panel display has been presented at ISCAS 2004 (Saas et al., 2004). Although the presented solution had some limitations, it clearly demonstrated the possibilities regarding power consumption.

The proposed architecture implements as many components as possible in the digital domain as to minimize the effort in power and area for analog components. The presented DA converter is capable of driving the lines and therefore there is no need for analog line drivers. The digital registers consume significantly less energy than the analog counterpart. Although the new logic $L^{\prime}$ is slightly more complicated and one DAC is needed at each line, the power consumption is negligible with respect to the bias currents of the conventional line drivers.

Stepwise charging is realized by applying a digital control signal, which will switch the output to the next higher value for each time step until the desired voltage level is reached. To benefit from the possible power savings it is important to ensure that an intermediate voltage level is almost reached before the next clock edge. A significant part of the energy on the line can be reused by applying charge recovery. Large capacitors are used to temporarily deliver energy and to recover the charge when driving analog output voltages.
There is one large source capacitor $C_{\mathrm{s}}$ per reference voltage. It is charged by reference voltage sources to the appropriate voltage level during the reset period. Afterwards the whole DAC is powered from the capacitors. As there is only one reference voltage generator per display, the source capacitors could be realized as external elements.

The output is discharged stepwise by connecting the load to the appropriate reference voltages. Thus every clock cycle the output voltage is decreased by $V_{\text {full }} / N$. When the output is connected to the next lower reference voltage level some of the charge is transferred back to $C_{\mathrm{s}}$ and the corresponding reference voltage across $C_{\mathrm{s}}$ is nearly restoring the initial value. Of course, there can be no energy transferred to the highest voltage level. The amount of energy taken from this reference voltage is prorated on the charging and discharging of the single s.pdf. In the following reset the losses are compensated by connecting $C_{\mathrm{s}}$ to the appropriate reference voltage. It can easily be calculated that in theory all but the highest source capacitors $C_{\mathrm{s} \max }$ recover charge up to their original voltage value. The energy which has been dissipated and thus has to be replaced in $C_{\mathrm{s} \max }$ is given by

$E_{\mathrm{s} \max }=\frac{V_{\text {full }}}{N} \cdot V_{\max } \cdot C_{\mathrm{L}}$

where $V_{\max }$ is the voltage the line was charged up to and $C_{\mathrm{s} \text { max }}$ the corresponding source capacitor.

Although this driver has been presented for a 3 bit resolution, it can easily be scaled to 4 bit.

\section{Increasing bit depth}

Up to date color displays for mobile applications require at least a 6 bit resolution per color to be competitive. To obtain a full dynamic color resolution, a bit depth of 8 bit is required. Higher bit depths in a display are usually used for calibration reasons and are obsolete when other means of color calibration are provided.

The scaling of the driver proposed in Sect. 2 is not a suitable approach to obtain a higher dynamic resolution as this would increase the number of supply capacitances and low impedance voltage sources thus leading to an unacceptable overhead. In this work a hybrid solution is proposed. Stepwise charging is used to generate an approximated output level corresponding to the 4 most significant bits (MSB) and a second stage is evaluating the 4 least significant bits (LSB) to obtain an accurate voltage level. Due to a number of systematic offset errors some means of calibration has to be provided.

\subsection{Adding a defined charge}

As the load capacitance of the driver is constant and linear with very good approximation, a defined voltage difference 


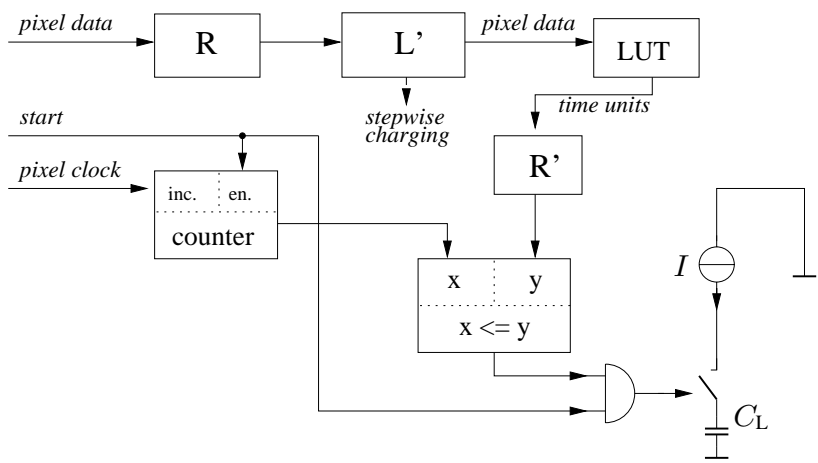

Fig. 2. Architecture of the full dynamic resolution driver.

can be set by adding a certain amount of charge.

$\Delta V=\frac{Q}{C}$

Assuming a constant charging current, the voltage difference is determined by the on time of the current. Even in the case of a not perfectly constant current the time values to obtain a correct pixel value can be easily obtained by calibration.

The final voltage levels are not taken from a reference voltage but are locally evaluated in the driver. The proposed solution exhibits the advantage of a reduced number of reference voltage lines from the reference voltage generator to the source drivers. This goes very well along with the industry standard proposed by National Semiconductor which also reduces the number of $V_{\text {ref }}$ lines by generating intermediate levels locally in the driver (McCartney, 2004).

\subsection{The current sources}

Current sources are typically realized by a current mirror. In the present application additional switching requirements exist which are not covered by the standard CMOS current mirror; a modified current mirror is introduced.

The reference currents are generated by a standard bias generator. This current has to be mirrored several times according to the number of source drivers. As the pixel-value is in general different for each column, each of the current mirrors has to be independently switchable. This is not possible with a standard CMOS mirror, as the switching transistor would introduce a significant mirror error during operation. The proposed circuit is shown in Fig. 3.

Analytical proof of the proper function of the proposed circuit is only carried out for equally sized transistors $T_{1}, T_{2}, T_{3}$ and $T_{4}$. Nevertheless, the current mirror also works for other transistor sizes which may be desirable to avoid devices in non-saturation region. Please note that channel length modulation is neglected. For $T_{2}$ and $T_{3}$ in saturation, it is obvious, that $I_{\mathrm{ds} 2}$ equals $I_{\mathrm{ds} 3}$. However, it also holds true for $(W / L)_{2}=(W / L)_{1}$.

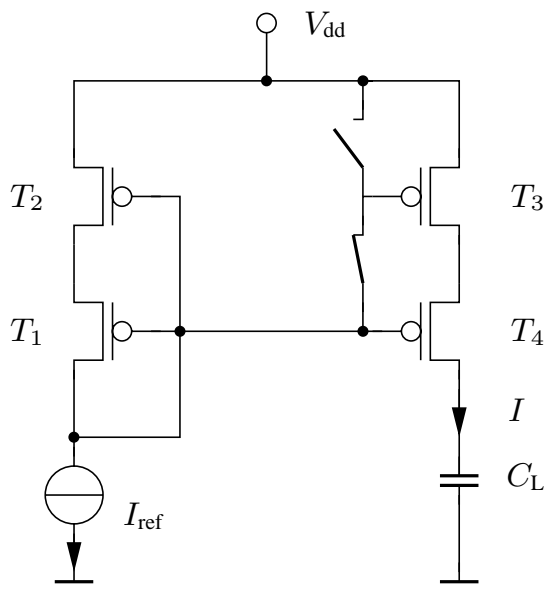

Fig. 3. Schematic of a switchable current source.

The conditions for a PMOS transistor to be in saturation are

$V_{\mathrm{gs}}<V_{\mathrm{th}, \mathrm{P}}$

$V_{\mathrm{ds}} \leq V_{\mathrm{gs}}-V_{\mathrm{th}, \mathrm{P}}$

For $T_{1}$ Eq. (4) is satisfied, otherwise it would be in cut-off mode, which is impossible as long as $I_{\mathrm{sd} 1}=I_{\text {ref }} \geq 0$. Equation 5 is satisfied, too, since $V_{\mathrm{ds} 1}=V_{\mathrm{gs} 1}$ and $V_{\mathrm{th}, \mathrm{P}}<0$. Obviously, $T_{2}$ is in non-saturation, because its current equals $I_{\mathrm{sd} 1}$, but $V_{\mathrm{gs} 2} \neq V_{\mathrm{gs} 1}$ and different $V_{\mathrm{gs}}$ for identical transistors that are both saturated lead to different currents. Thus, $T_{1}$ is in saturation, $T_{2}$ in non-saturation mode. Note that all voltages of $T_{1}$ and $T_{2}$ are independent of the supply voltage $V_{\mathrm{dd}}$. For $T_{4}$, Eq. 5 is satisfied if

$V_{\mathrm{ds} 4} \leq V_{\mathrm{gs} 4}-V_{\mathrm{th}, \mathrm{P}}$

or

$V_{\mathrm{ds} 4}+V_{\mathrm{ds} 3} \leq V_{\mathrm{gs} 3}-V_{\mathrm{th}, \mathrm{P}}$.

The sum of $V_{\mathrm{ds} 4}$ and $V_{\mathrm{ds} 3}$ depends on the voltage on the load capacitance and on $V_{\mathrm{dd}}$. Thus, we must apply an adequate supply voltage. $T_{4}$ is in saturation mode and $T_{3}$ in nonsaturation mode as explained above. The drain currents of $T_{3}$ and $T_{4}$ have to be equal:

$I_{\mathrm{ds} 3}=I_{\mathrm{ds} 4}$.

Plugging in the transistor equations gives

$K_{\mathrm{p}}\left(V_{\mathrm{gs} 3}-V_{\mathrm{th}, \mathrm{P}}-\frac{V_{\mathrm{ds} 3}}{2}\right) V_{\mathrm{ds} 3}=\frac{K_{p}}{2}\left(V_{\mathrm{gs} 4}-V_{\mathrm{th}, \mathrm{P}}\right)^{2}$

Replacing $V_{\mathrm{gs} 4}$ in Eq. 9 leads to an equation that demonstrates that $V_{\mathrm{ds} 3}$ only depends on $V_{\mathrm{gs} 3}$ and $V_{\mathrm{th}, \mathrm{P}}$. In the same way $V_{\mathrm{ds} 2}$ only depends on $V_{\mathrm{gs} 2}$ and $V_{\mathrm{th}, \mathrm{P}}$. Since the $V_{\mathrm{gs}}$ of $T_{2}$ and $T_{3}$ are equal, their $V_{\mathrm{ds}}$ have to be equal, too. Because both transistors are in non-saturation region and the same size, both currents are also equal. Thus, it is proved that $I=I_{\text {ref }}$, and the proposed circuit is a current mirror. 
Please note that the proposed circuit has no improvement over the standard mirror with respect to the mirror error, but it has additional switching capabilities.

To fulfill timing requirements in the display, a minimum charging current has to be applied. In the worst case the load capacitance has to be charged a full MSB-step within one cycle. Charging time for a $17 \mu \mathrm{s}$ cycle may last up to $4 \mu \mathrm{s}$.

$I_{\min }=\frac{\frac{1}{16} V_{\max } C_{\mathrm{L}}}{t_{\max }} \approx 16 \mu \mathrm{A}$

On the other hand, there is also an upper bound due to accuracy requirements. As the time $t$ is derived from a clock signal, there are quantization errors in the charging time $t$.

$I_{\text {max }}=\frac{. p d \text { filon }_{\text {max }} \cdot C_{\mathrm{L}}}{t_{\min }} \approx 78 \mu \mathrm{A}$

where .pdfilon max $_{\text {in }}$ is the maximum acceptable error.

\subsection{Preset values for the lookup table}

Due to variations in the current with respect to the output voltage the lookup table has to hold a number of charging time units for each of 256 possible output values. Nevertheless, this small amount of memory is negligible in comparison to the analog parts of the driver. The values in the lookup table can be easily obtained by color calibration of the display and set by a software tool.

However, calculating this values systematically delivers a preset for the table. For this purpose, a reasonable model of the current is required. The current versus load voltage is not linear. However, since only small changes of the output value due to the LSBs are of interest $I$ can be approximated with good accuracy by

$I=I_{0}-\alpha \Delta V$,

where $I_{0}$ is the current at LSB 0000 and $\alpha$ the negative slope. $\alpha$ can be easily derived from the current values of two adjacent MSB values.

As we know that

$C_{\mathrm{L}} \Delta V=\int_{0}^{t} I d t$

we derive

$\Rightarrow \Delta V+\frac{\alpha}{C_{\mathrm{L}}} \int_{0}^{t} \Delta V d t=\frac{I_{0} t}{C_{\mathrm{L}}}$

and

$\frac{d}{d t} \Delta V+\frac{\alpha}{C_{\mathrm{L}}} \Delta V=\frac{I_{0}}{C_{\mathrm{L}}}$.

The solution to this differential equation is given by

$\Delta V=\frac{I_{0}}{\alpha}\left(1-e^{-\frac{\alpha}{C_{\mathrm{L}}} t}\right)$.

Therefore, the charging time is given by

$t=-\frac{C_{\mathrm{L}}}{\alpha} \ln \left(1-\alpha \frac{\Delta V}{I_{0}}\right)$.
Equation 17 gives a good approximation for the charging time.

In addition, the delay between the switching signal and the output current has to be taken into account. On the one hand, there is a significant delay between the transition of the control signal and the current reaching its nominal value. On the other hand the current is not cut off immediately after the control signal returns to the off state. These delays have to be considered and the time values have to be adjusted accordingly.

\subsection{Fine adjustment and Gamma correction}

Today's liquid crystal flat panel displays need extensive gamma correction due to nonlinearities in the voltage - transmission curve. The properties of the crystal vary over time, so the display needs to be calibrated if accurate color reproduction is an issue. Although the nonlinearities for the optical emission with respect to the current are much weaker in an OLED display, there is still the need for calibration. In the proposed circuit, gamma correction and calibration can be carried out rather easy by adjusting the values in the lookuptable. This can be done during runtime by a software tool.

\subsection{Energy performance of the LSB part}

As the energy performance of the stepwise MSB part is well understood (Saas et al., 2004), this section will only cover the additional dissipation due to the LSB part. The generation of the reference currents and the reference part of the mirror supply a significant number of source drivers and can be neglected.

The energy taken from the supply by a single source driver during charging is given by

$E_{\text {current source }}=V_{\mathrm{dd}} \cdot I_{\mathrm{ref}} \cdot t=C_{\mathrm{L}} \cdot V_{\mathrm{dd}} \cdot \Delta V$

Please note that the supply voltage $V_{\mathrm{dd}}$ is higher than the maximum output voltage. Table I shows the calculated values for some LSB values. A significant part of the energy delivered by the LSB part is stored onto the load capacitance $C_{\mathrm{L}}$.

$E_{\text {additional }}=C_{\mathrm{L}} \cdot \Delta V \cdot V_{\mathrm{MSB}}-\frac{1}{2} C_{\mathrm{L}} \cdot \Delta V^{2}$

This energy can be recovered during stepwise discharging and reduces energy losses in the MSB part.

\subsection{Voltage follower based solutions}

Solutions based on a voltage follower have also been investigated (Saas and Francke, 2004). They were not competitive neither in terms of energy consumption nor in robustness and calibration possibilities and are therefore not mentioned in this article. 
Table 1. Calculated energy for selected LSBs.

\begin{tabular}{c||c|r|r}
\hline $\begin{array}{c}\text { LSB } \\
\text { code }\end{array}$ & $\begin{array}{c}\text { Desired voltage } \\
\text { level } / \mathrm{mV}\end{array}$ & $\begin{array}{c}\text { Ideal charging } \\
\text { time } / \mathrm{ns}\end{array}$ & $\begin{array}{r}\text { Delivered } \\
\text { Energy } / \mathrm{pJ}\end{array}$ \\
\hline \hline 0000 & 0000.00 & 0.0 & 0.000 \\
\hline 0001 & 0019.53 & 195.3 & 31.25 \\
\hline 0101 & 0097.66 & 976.6 & 156.25 \\
\hline 1011 & 0214.84 & 2148.4 & 343.74 \\
\hline 1111 & 0292.97 & 2929.7 & 468.75 \\
\hline
\end{tabular}

Table 2. Simulation result for the MSB part.

\begin{tabular}{l|c|c|c}
\hline & conventional $C V^{2}$ & $\frac{1}{N} C V^{2}$ & simulation \\
\hline 3 bit driver & $294 \mu \mathrm{W}$ & $42.0 \mu W$ & $46.3 \mu \mathrm{W}$ \\
4 bit driver & $294 \mu \mathrm{W}$ & $19.6 \mu W$ & $22.4 \mu \mathrm{W}$ \\
\hline
\end{tabular}

\section{Simulation results}

Simulations have been carried out using a $180 \mathrm{~nm}$ CMOS process. In addition some high voltage devices where used due to the required output voltage range. These technologies are not used in real display production, but they are sufficiently similar to allow a good estimation of the possibilities of this new concept.

\subsection{MSB part}

The power consumption of the improved stepwise driver scales very well with the increased number of s.pdf. The simulation results as well as theoretical estimations are listed in Table 2. As expected the energy dissipation is even smaller than that of the three bit driver due to the smaller step-size. The gain of both with respect to the conventional solution is obvious.

Further elaborations on the simulation results of the stepwise driver can be found in Saas et al. (2004).

\subsection{LSB part}

The simulations results of the LSB part are shown in Table 3. Results are listed for all LSB values at some representative MSB values. Although results are slightly different for different MSB values, it can be seen, that simulations correspond very well with the estimations made in Sect. 3.5. The average values listed in the table have been calculated assuming an equally distribution of pixel values. Additional simulations have been carried out to evaluate power consumption due to leakage currents. The total power consumption due to static parasitic effects was in the worst case $0.0034 \mathrm{pJ}$ over a whole cycle. This small amount is negligible with respect to the dynamic power consumption.
Table 3. Simulated delivered energy for charging LSBs in pJ.

\begin{tabular}{c||c|c|c||c}
\hline LSB & MSB 1000 & MSB 0000 & MSB 1111 & Average \\
\hline \hline 0000 & 0.00 & 0.00 & 0.00 & 0.00 \\
\hline 0001 & 35.31 & 34.91 & 35.44 & 35.22 \\
\hline 0010 & 66.58 & 66.68 & 66.83 & 66.70 \\
\hline 0011 & 97.84 & 97.56 & 97.40 & 97.60 \\
\hline 0100 & 129.09 & 129.31 & 128.75 & 129.05 \\
\hline 0101 & 160.32 & 160.17 & 160.09 & 160.19 \\
\hline 0110 & 191.11 & 191.91 & 191.40 & 191.47 \\
\hline 0111 & 222.75 & 222.74 & 222.71 & 222.73 \\
\hline 1000 & 253.95 & 253.56 & 253.99 & 253.83 \\
\hline 1001 & 285.14 & 285.27 & 285.26 & 285.22 \\
\hline 1010 & 316.31 & 316.96 & 316.51 & 316.60 \\
\hline 1011 & 347.48 & 347.76 & 347.74 & 347.66 \\
\hline 1100 & 379.12 & 379.43 & 378.96 & 379.17 \\
\hline 1101 & 410.61 & 410.22 & 410.16 & 410.33 \\
\hline 1110 & 441.74 & 441.87 & 441.34 & 441.65 \\
\hline 1111 & 472.86 & 472.63 & 472.49 & 472.66 \\
\hline \hline Average & 238.14 & 238.19 & 238.07 & 238.13 \\
\hline & & & & \\
\hline
\end{tabular}

As explained in Saas et al. (2004) no conventional driver has been designed and simulated as a reference. It has been shown the the proposed architecture minimizes energy consumption to about $10 \%$ of a theoretical lower bound. The simulation results for the improved driver with a dynamic resolution of 8 bit show the power consumption is in the same order of magnitude. The average power consumption of $238.13 \mathrm{pJ}$ translates into $14 \mu \mathrm{W}$ for the clock cycle of $17 \mu \mathrm{s}$. Even if the additional charge-recovery during discharging is not taken into account, the new driver still consumes significantly less than the conventional solution.

\section{Conclusions}

The performance of the proposed architecture proves that stepwise charging is well suited to reduce power dissipation in the source driver in a full color resolution display. The energy consumption can be significantly reduced while preserving accuracy for the voltage levels and meeting timing constraints for typical display resolutions.

\section{References}

Athas, W., N.Tzartzanis, Mao, W., Peterson, L., Lal, R., Chong, K., Moon, J.-S., Svenson, L., and Bolotski, M.: The Design and Implementation os a Low-Power Clock-Powered Microprocessor, Journal od Solid State Circuits, 35, 1561-1570, 2000. 
McCartney, R.: A third generation timing Controler and column driver architecture using point-to-point differential signaling, in: Proceedings of SID Symposium, Seattle, 2004.

Saas, C. and Francke, J.: Increasing Bit Depth in Low Power DAConverters for Display Applications, Tech. Rep. TUM-LNS-TR04-7, Munich University of Technology, 2004.

Saas, C. and Nossek, J.: Resonant Multistage Charging of Dominant Capacitances, in: Proceedings of PATMOS, Sevilla, 2002.
Saas, C., Wróblewski, A., and J.A.Nossek: Low-Power DAConverters for Display Applications using Stepwise Charging and Charge Recovery, in: Proceedings of ISCAS, Vancouver, 2004.

Ziesler, C., Kim, S., and Papaefthymiou, M.: A Resonant Clock Generator for Single-Phase Adiabatic Systems, ISLPED, 2001. 